\title{
Supracategorical fear information revealed by aversively conditioning multiple categories
}

\author{
Seth M. Levine ${ }^{1,2, * 凶}$, Miriam Kumpf ${ }^{2, *}$, Rainer Rupprecht ${ }^{2}$, and Jens V. Schwarzbach ${ }^{2}$ \\ ${ }^{1}$ Department of Cognitive and Clinical Neuroscience, Central Institute of Mental Health, Medical Faculty Mannheim, Heidelberg University, 68163 Mannheim, Germany \\ ${ }^{2}$ Department of Psychiatry and Psychotherapy, University of Regensburg, 93053 Regensburg, Germany
}

\begin{abstract}
Fear-generalization is a critical function for survival, in which an organism extracts information from a specific instantiation of a threat (e.g., the western diamondback rattlesnake in my front yard on Sunday) and learns to fear-and accordingly respond to-pertinent higher-order information (e.g., snakes live in my yard). Previous work investigating fear-conditioning in humans has used functional magnetic resonance imaging (fMRI) to demonstrate that activity-patterns of stimuli from an aversively-conditioned category (CS+) are more similar to each other than those of a neutral category (CS-). Here we designed a three-phase (i.e., baseline, conditioned, extinction) experiment using fMRI and multiple aversively-conditioned categories to ask whether we would find only similarity increases within the CS+ categories or also an increase in similarity between the CS+ categories. Using representational similarity analysis, we correlated a set of models to activity-patterns underlying several regions of interest and found that, following fearconditioning, between-category and within-category similarity increased for the CS+ categories in the superior frontal gyrus (SFG) and the right temporal pole (rTP). Activity patterns in the object-selective lateral occipital cortex tended to prefer the semantic model, regardless of the experimental phase. These results advance prior pattern-based neuroimaging work by exploring the effect of aversively-conditioning multiple categories and indicate an extended role for the SFG and rTP in potentially linking discrete information or abstractly representing supracategorical information during fear-learning for the purpose of proper generalization.
\end{abstract}

Keywords: aversive-learning | fear-conditioning | fMRI | multivariate pattern analysis | representational similarity analysis

区 Correspondence: seth.levine@zi-mannheim.de *Equal contribution

\section{Introduction}

Fear-learning is an essential process for an organism's survival, as it allows for a reasonable response to environmental threats. Classically, this process has been studied through fear-conditioning, a form of Pavlovian conditioning, in which an organism is exposed to a neutral stimulus (e.g. a picture of a dandelion) that is paired with an aversive, unconditioned stimulus (US, e.g., a mildlypainful electric shock); following a sufficient number of such pairings, the neutral stimulus is rendered a conditioned stimulus (CS+) and, even absent the US, yields the same physiological fear-response in the organism $[1,2]$.

This basic process gives rise to the higher-order process of fear-generalization. Recognizing that an entity in the environment might be dangerous based on its similarity to other previously-encountered entities is a critical extension of fear-learning. With respect to the previous example, feargeneralization could result in a fear-response upon seeing other plants besides dandelions. While the physiological process of fear-generalization may prove helpful in some situations, it stands to reason that a faulty generalization process could explain a variety of psychiatric disorders that are characterized by elevated levels of fear or anxiety $[3,4]$. As anxiety disorders have a lifetime prevalence of $16.6 \%$ [5], investigating the neural mechanisms of feargeneralization in humans is of particular interest to the translational neuroscience community.

Numerous neuroimaging studies have demonstrated that the amplitude of the blood-oxygen-level-dependent (BOLD) signal evoked by a CS+ differs from that evoked by a neutral stimulus (CS-) in various regions of the human brain (see [6] for a review). In terms of multivariate pattern analysis (MVPA) [7], recent work has shown increased similarity between activity patterns evoked by a CS+ and those evoked by images of spiders in the right anterior temporal lobe of humans with arachnophobia [8]. Other studies employing pattern-based analyses have shown that the activity patterns evoked by a CS+ in the insular cortex bear a closer resemblance to those evoked by the US [9] or that the activity patterns representing stimuli belonging to the same semantic category (e.g., animals or tools) in object-selective cortex are more similar to each other after some elements of the category have been paired with an electric shock [10]. Following especially from this last example, one could surmise that, given the increased withincategory similarity, the human brain has the cognitive ability to generalize a specific, conditioned fear to a superordinate group. We were interested in building on this finding and investigating whether fear-conditioning could also elicit increased pattern similarity between semantically unrelated categories.

To this end, we conducted a three-phase experiment (i.e., baseline, conditioned, extinction) in which participants viewed images from three categories (i.e., animals, produce, and tools) while lying in the MR scanner. During the conditioned phase, $50 \%$ of the images from two of the categories were paired with mildly aversive electric shocks, while the third category remained neutral throughout the entire experiment. Within the framework of representational similarity analysis (RSA) [11], we used a set of models to explain how the pattern similarity 
of the stimuli might change as a function of fearconditioning. Specifically, we were interested in whether we would only see increases in similarity within the CS+ categories or (also) between the CS+ categories. The models make different assumptions about the underlying operating principles of fear-generalization, with respect to categorical knowledge, and can therefore reveal differences in information processing of distinct brain regions that are involved in learning, affect, and semantics.

\section{Methods}

\section{Participants}

Twenty-one participants (6 males, 15 females; mean age $=25.2$ yrs, age range $=20-30$ yrs) were recruited from the local community. Participants had no current neuropsychiatric diagnoses, were not taking any psychotropic medication, and provided written informed consent before taking part in the study. All experimental procedures were approved by the ethics committee of the University of Regensburg and complied with the Declaration of Helsinki.

\section{Stimuli}

Stimuli used in the experiment comprised three distinct categories: animals, produce (i.e., fruits/vegetables), and tools. Thirty unique images from the three categories were obtained from multiple sources, either contributed by other researchers [12] or freely available online [13, 14] (www.pexels.com, www.unsplash.com). We avoided using potentially threatening images (e.g., knives/spiders) to lessen arousal-based effects.

The visual stimuli were presented with A Simple Framework [15], built on the Psychophysics toolbox [16] and MATLAB R2015b (Mathworks, Natick, USA), and cast onto a semitransparent screen behind participants using an LED projector (PROPixx, VPixx Technologies Inc., Saint Bruno, Canada) at a frame rate of $60 \mathrm{~Hz}$ and a resolution of $1024 \times$ 768 pixels.

Electrical stimuli (duration $=2 \mathrm{~ms}$ ) were delivered via a DS7A current stimulator (Digitimer Limited, Letchworth Garden City, UK), the timing of which was controlled by ASF and an Arduino® (Arduino SA, Chiasso, Switzerland).

\section{Experimental design}

\section{Pre-scan protocols}

Participants first completed the German version of the StateTrait Anxiety Inventory [17], which are two questionnaires each containing 20 self-reported items used to assess state and trait anxiety on a 4-point Likert scale.

Next, participants completed a threshold-acquisition session, in which they applied electric shocks to their left wrists, while increasing the amperage, until they found a near-painful sensation that they considered tolerable but unpleasant. The individualized amperages $($ mean $=11.13$ $\mathrm{mA}$, range $=1.5-29.0 \mathrm{~mA}$ ) obtained from this session were used during the conditioned phase of the main experiment.
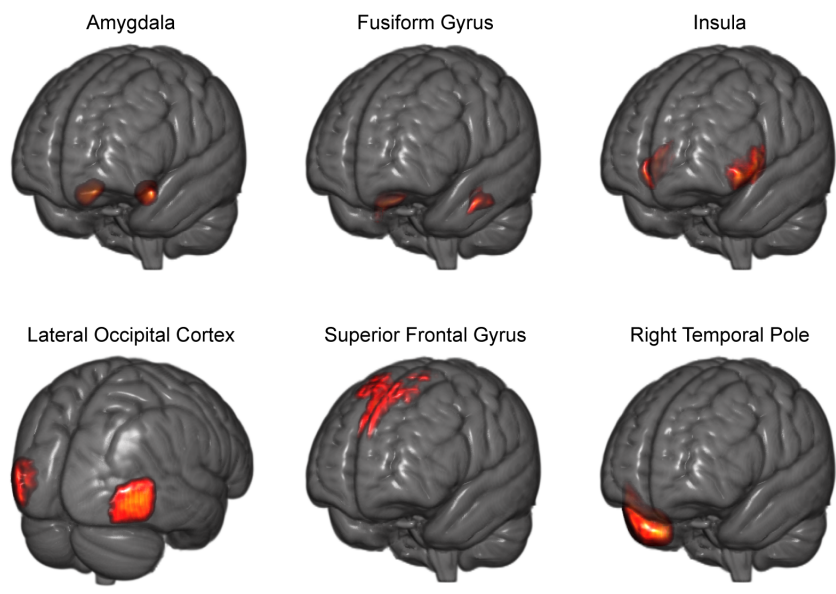

Fig. 1. Regions of interest (ROIs) used in the current study based on previous fear-learning investigations that demonstrated conditioning-based modulations of multivoxel patterns. See section Region-of-interest definition for details about the $\mathrm{ROI}$ selection and definition.

\section{Main experiment}

The main experiment contained three phases: baseline, conditioned, and extinction. Each phase followed the same event-related protocol, with the exception that participants received electric shocks during the conditioned phase. During a given phase, participants viewed 90 stimuli in total (i.e., 30 stimuli from each category). To prevent discomfort, rather than having participants undergo one long run (per phase) with 90 stimulus presentations, each phase was split into three shorter runs, in which we presented 30 stimuli (i.e., 10 stimuli per category) per run. A given run thus contained 30 trials; each trial was $3 \mathrm{~s}$ in duration $(2 \mathrm{~s}$ of stimulus presentation $+1 \mathrm{~s}$ of fixation), followed by a temporallyjittered intertrial interval of $6+X \mathrm{~s}$, with $\mathrm{X} \sim \operatorname{geom}(0.3)$, truncated at $10 \mathrm{~s}$, yielding possible intertrial intervals of 6 to $10 \mathrm{~s}$ in steps of $0.5 \mathrm{~s}$. Each run started with a $12 \mathrm{~s}$ fixation period, to account for T1 saturation, and ended with a $12 \mathrm{~s}$ fixation period to capture the BOLD response from the final trial. In total, a given run lasted between $4 \mathrm{~min} 48 \mathrm{~s}$ and 6 min $44 \mathrm{~s}$, depending on the intertrial jitter, with an average run length of approximately $5 \min 25 \mathrm{~s}$. A black fixation dot was always present in the center of the screen.

During all runs, to ensure that participants were paying attention to the visual stimuli, we asked them to perform a one-back task, in which they indicated via button-press whether the image they viewed on a given trial was from the same category (right index finger response) or a different category (right middle finger response) as the image from the previous trial. Participants' responses were recorded starting from the onset of the stimulus presentation to the end of the $1 \mathrm{~s}$ fixation period that followed the stimulus.

Additionally, during the conditioned phase, electrical stimulation was administered at the offset of stimulus presentation for $50 \%$ of images that belonged to two of the three categories. In each run of the conditioned phase, there were thus 10 electrical shocks (i.e., 5 shocks per conditioned category); the first presentations of exemplars from conditioned categories co-terminated with electric 


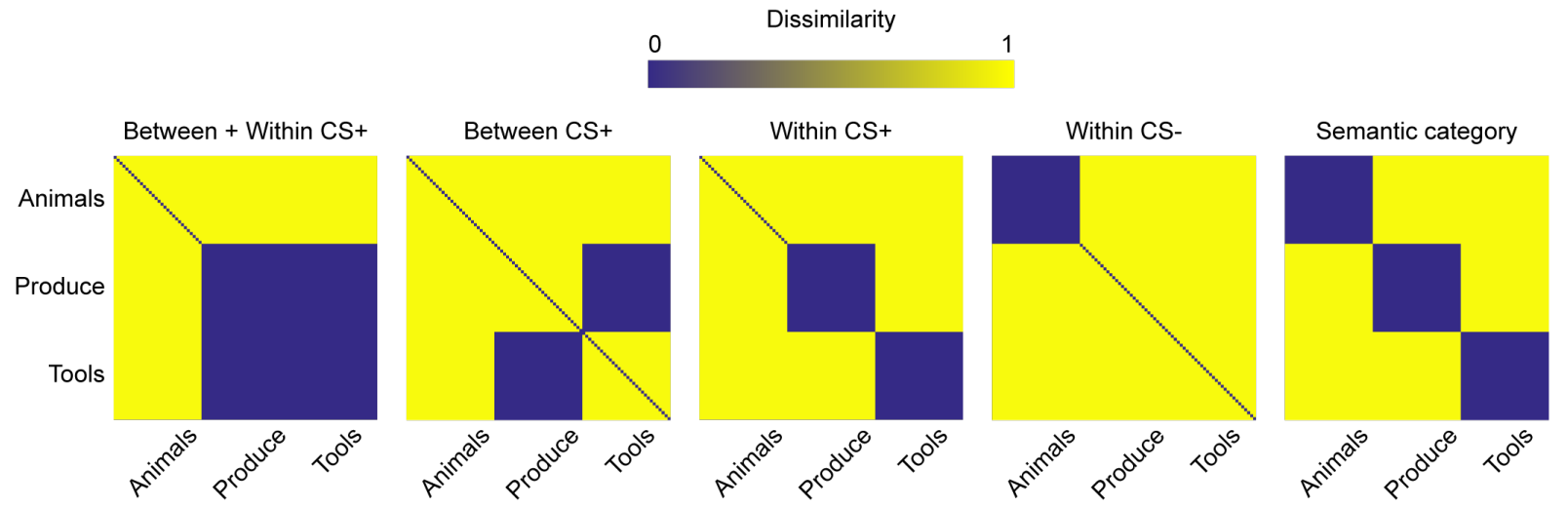

Fig. 2. A visual depiction of the five model dissimilarity matrices (DSMs) used in the representational similarity analysis. Each $90 \times 90$ matrix reflects the binarized dissimilarity between each pair of the 90 stimuli that a participant viewed during a given phase of the experiment. Together the five DSMs represent distinct hypotheses regarding the relationship among the stimuli from three distinct semantic categories (i.e., 30 animals, 30 fruits/vegetables, and 30 tools), which we used to test whether aversive-learning would yield increases in within-category or between-category similarity (or both) at each of the six ROls. As the models were specific to certain participants (depending on which categories were fear-conditioned), depicted here are example DSMs from a participant for whom produce and tools served as the two CS+ categories. One-third of the participants were conditioned to animals and produce, one-third to animals and tools, and one-third to produce and tools. For all participants, the rightmost DSM (semantic category) was identical and served as a control model.

shocks, to ensure that the conditioning was sufficiently re-instated between runs. The remaining four shocks per category (per run) were pseudorandomized across the remaining presentations of the stimuli of those categories. Of the 21 participants, seven were conditioned to animals and produce, seven were conditioned to animals and tools, and seven were conditioned to produce and tools.

\section{Neuroimaging data acquisition}

The neuroimaging experiment was carried out at the University of Regensburg, Germany using the 3T researchdedicated MR scanner (Magnetom Prisma, Siemens, Erlangen, Germany) and a 64-channel head coil. Functional images were acquired with a T2*-weighted EPI sequence (64 slices per volume, acquired with a multiband factor of $4[18,19]$, field of view $(\mathrm{FOV})=192 \times 192 \mathrm{~mm} 2$, isotropic voxel resolution (VR) of $2 \times 2 \times 2 \mathrm{~mm} 3$, no interslice gap, repetition time $(\mathrm{TR})=2000 \mathrm{~ms}$, echo time $(\mathrm{TE})=30 \mathrm{~ms}$, flip angle $(\mathrm{FA})=75^{\circ}$ ). Four dummy scans from the beginning of each functional run were acquired to account for signal saturation. For a given run of the main experiment, we acquired between 144 to 202 volumes, depending on the intertrial jitter (see section Main experiment).

For co-registration of the functional images to highresolution anatomical images, we acquired 160 slices of a T1-weighted magnetization-prepared rapid gradient-echo (MPRAGE) sequence $(\mathrm{FOV}=256 \times 256 \mathrm{~mm} 2, \mathrm{VR}=0.9766$ $\times 0.9766 \times 1 \mathrm{~mm} 3, \mathrm{TR}=1910 \mathrm{~ms}, \mathrm{TE}=3.67 \mathrm{~ms}, \mathrm{FA}=9^{\circ}$ ) for each participant.

\section{Data analysis}

Neuroimaging data were analyzed with the FMRIB Software Library (FSL) [20] and the CoSMoMVPA toolbox [21] for MATLAB.

\section{Preprocessing}

Preprocessing of the functional data included removal of nonbrain tissue [22], slice time correction, motion correction with respect to the middle volume of each run (using 6 degrees of freedom and trilinear interpolation), and highpass filtering (cutoff $=100 \mathrm{~s}$ ). No spatial smoothing was performed. We co-registered each participant's functional data to the corresponding high-resolution structural scan using 7 degrees of freedom [23] and then to standard space (i.e., MNI152 2mm) using 12 degrees of freedom.

\section{First-level modeling}

For each run of the experiment, time-course data from each voxel were analyzed using the general linear model (GLM) containing 30 regressors of interest (i.e., one regressor per image presented to the participant) and six motion correction parameters (i.e., 3 translations, 3 rotations) modeled as regressors of non-interest. Hemodynamic response functions were generated by convolving the regressors of interest with gamma functions using FSL's default parameters $\phi=0 \mathrm{~s}, \sigma$ $=3 \mathrm{~s}$, mean lag $=6 \mathrm{~s}$ ). The resulting t-scores of the beta weights per regressor were concatenated across the three runs of an experimental phase, resulting in 90 whole-brain t-score maps (per phase), reflecting the 90 stimuli that a participant viewed.

\section{Region-of-interest definition}

Regions-of-interest (ROIs) comprised the amygdala, posterior fusiform gyrus, insula, inferior lateral occipital cortex (LOC), superior frontal gyrus (SFG), and right temporal pole (rTP), which we selected based on previous neuroimaging studies that similarly combined forms of MVPA [7] with aversive-learning [8-10, 24]. The ROIs were defined using the Harvard-Oxford probabilistic (sub)cortical atlas $[25,26]$ and contained voxels that belonged to the given region with a probability greater than 0.5 (see Fig. 1).

\section{Representational similarity analysis}

Within the six ROIs, we were mainly interested in determining whether, following fear-conditioning, activitypatterns would become more similar between conditioned 
categories or only within conditioned categories. To this end, we constructed five $90 \times 90$ model dissimilarity matrices (DSMs) that each contained zeros (indicating similarity between two stimuli) and ones (indicating dissimilarity between two stimuli). The five model DSMs reflected the following stimulus relationships: between + within $\mathrm{CS}+$, between $\mathrm{CS}+$, within $\mathrm{CS}+$, within $\mathrm{CS}-$, and semantic similarity (the last two models served as controls). The DSMs involving conditioned stimuli were participantspecific (as different participants were conditioned to different categories); the semantic DSM was the same for all participants (see Fig. 2).

For a given participant, we computed the pairwise similarity of the 90 activity patterns within each ROI (per phase; all 270 patterns for a given ROI were meancentered) using (Pearson) correlation distance (i.e., 1 r), which yielded a neural DSM reflecting the underlying representational space of these 90 stimuli in the given ROI. We then Spearman correlated our five model DSMs with the neural DSMs of the six ROIs, for each phase. This procedure was carried out for all participants, and the resulting 90 correlations (per participant) were Fishertransformed and submitted to a three-way repeated-measures analysis of variance (ANOVA) containing factors ROI (6 levels $) \times$ Phase $(3$ levels $) \times$ Model $(5$ levels $)$ computed with SPSS ver. 25 (IBM Corp., Armonk, NY, United States). Ultimately, we wanted to determine whether there was a three-way interaction between these factors, which would indicate that different model DSMs explain activation patterns in different experimental phases for different ROIs. Additionally, because we were specifically interested in determining whether representational spaces were modulated as a function of fear-conditioning, we carried out paired ttests for each model, testing whether the resulting correlation differed between the conditioned phase and the baseline phase. Computing these five t-tests at each of the six ROIs yielded 30 statistical tests, which we accounted for with a False Discovery Rate (FDR) correction [27]).

\section{Anxiety correlations}

Lastly, we wanted to determine whether there was a relationship between the change in the explanatory value of the RSA models identified from the FDR-correction and the participants' STAI scores. To this end, we carried out a set of post-hoc correlations between the STAI results and the correlation changes (from baseline to conditioned), of the $\mathrm{Bw}+\mathrm{Wi}, \mathrm{Bw}, \mathrm{Wi}$, and CS- models in the $\mathrm{SFG}$ and the $\mathrm{Bw}+\mathrm{Wi}$ model in the rTP.

Table 1. Results from the three-factorial repeated-measures ANOVA

\begin{tabular}{lccc} 
& F-score & df1, df2 & p-value \\
\cline { 2 - 4 } Region & 91.9 & 5,100 & $8.42 \times 10^{-36}$ \\
Phase & 4.87 & 2,40 & 0.013 \\
Model & 10.6 & 4,80 & $6.08 \times 10^{-7}$ \\
Region $\times$ Phase & 3.96 & 10,200 & $6.03 \times 10^{-5}$ \\
Region $\times$ Model & 29.9 & 20,400 & $6.96 \times 10^{-67}$ \\
Phase $\times$ Model & 6.02 & 8,160 & $9.30 \times 10^{-7}$ \\
Region $\times$ Phase $\times$ Model & 1.98 & 40,800 & $3.65 \times 10^{-4}$ \\
\hline
\end{tabular}

\section{Results}

\section{Increase in similarity both within and between conditioned categories in the superior frontal gyrus and temporal pole}

In order to initially discern whether there were overall differences between the models, the 90 correlation values that we obtained from correlating the activity patterns from six regions with the five models at three different phases were Fisher transformed and submitted to a three-way repeatedmeasures ANOVA. This procedure supported the observation of general differences among regions $\left(\mathrm{F}_{5,100}=91.9, \mathrm{p}=8.42\right.$ $\left.\times 10^{-36}\right)$, phases $\left(\mathrm{F}_{2,40}=4.87 \mathrm{p}=0.013\right)$, and models $\left(\mathrm{F}_{4,80}=\right.$ $\left.10.6, p=6.08 \times 10^{-7}\right)$, in addition to regions yielding different correlations depending on the phase (Region $\times$ Phase: $F_{10,200}$ $\left.=3.96, \mathrm{p}=6.03 \times 10^{-5}\right)$, models performing differently depending on the region (Region $\times$ Model: $\mathrm{F}_{20,400}=29.9, \mathrm{p}=$ $6.96 \times 10^{-67}$ ), and models performing differently depending on the phase (Phase $\times$ Model: $F_{8,160}=6.02, p=9.30 \times 10^{-7}$ ). Most critically, the ANOVA also supported the observed three-way interaction in that different models performed differently at different experimental phases, depending on the region (Region $\times$ Phase $\times$ Model: $\mathrm{F}_{40,800}=1.98, \mathrm{p}=3.65 \times$ $\left.10^{-4}\right)$. See Figure 3 and Table 1 for an overview of the results.

As we were specifically interested in whether the performance of our models was modulated by fearconditioning, we carried out a series of tests on the difference between each model's correlation to a region's activity patterns during the conditioned phase compared to the baseline phase. This procedure revealed that, following fear-conditioning, the Between+Within $(\mathrm{Bw}+\mathrm{Wi}) \mathrm{CS}+$ model better explained the activity patterns within the superior frontal gyrus (SFG: $\mathrm{t}_{20}=6.08, \mathrm{p}=6.06 \times 10^{-6}$ ) and the right temporal pole (rTP: $\mathrm{t}_{20}=4.00, \mathrm{p}=6.99 \times 10^{-4}$ ). The only other models whose corresponding tests surpassed the FDR-corrected threshold of 0.0042 were the Between (Bw) CS+ model, the Within (Wi) CS+ model, and the Within CS(CS-) model within respect to activity patterns in the SFG $\left(\mathrm{t}_{20}=5.30, \mathrm{p}=3.50 \times 10^{-5} ; \mathrm{t}_{20}=3.49, \mathrm{p}=0.0023 ;\right.$ and $\mathrm{t}_{20}=-3.53, \mathrm{p}=0.0021$, respectively). Although the overall pattern of results for the Insula were qualitatively similar to those of the SFG and rTP, the change in performance of the $\mathrm{Bw}+\mathrm{Wi}$ model at the Insula did not surpass the FDRcorrected threshold $\left(\mathrm{t}_{20}=2.71, \mathrm{p}=0.013\right)$. See Figure 3 for an overview and Table 2 for a full list of the corresponding results.

Additionally, following fear-conditioning, the $\mathrm{Bw}+\mathrm{Wi}$ model outperformed the Bw model (which was the next best model $)$ in the SFG $\left(\mathrm{t}_{20}=2.34, \mathrm{p}_{\mathrm{unc}}=0.0296\right)$ but not in the rTP $\left(t_{20}=1.198, p_{\text {unc }}=0.1225\right)$. However, in both the SFG and the rTP, the $\mathrm{Bw}+\mathrm{Wi}$ model yielded a greater change from the baseline phase to the conditioned phase as compared to the $\mathrm{Bw}$ model $\left(\mathrm{t}_{20}=2.43, \mathrm{p}_{\text {unc }}=0.0245 ; \mathrm{t}_{20}=2.42, \mathrm{p}_{\text {unc }}=\right.$ 0.0253 , respectively). 


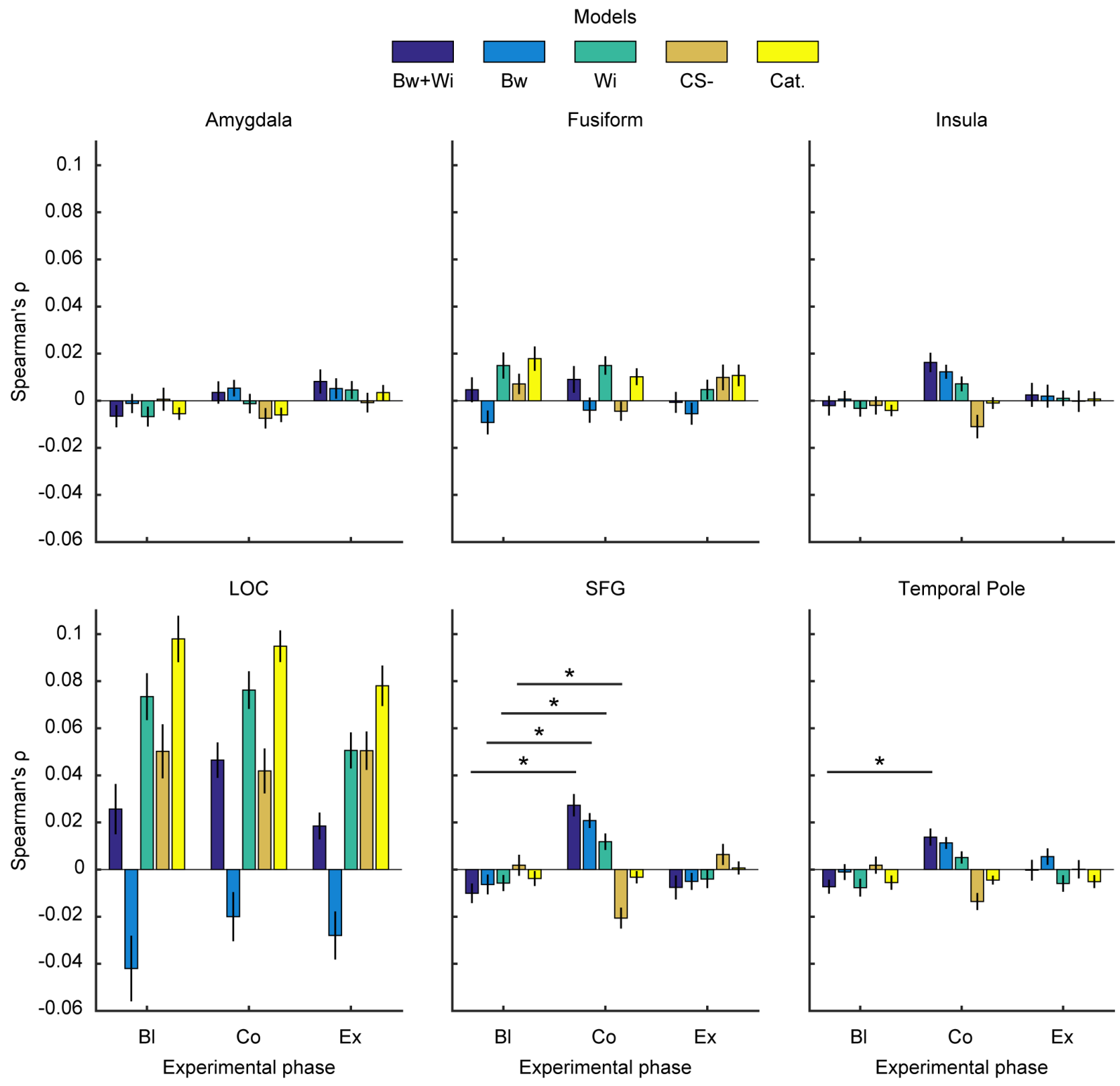

Fig. 3. Spearman correlations of the activity patterns from each ROI (Fig. 1) with the five model DSMs (Fig. 2) at the three phases of the experiment. The resulting correlations exhibited a three-way interaction in that different models explained activity patterns of different ROIs differently in different experimental phases (as supported by a three-factorial repeated-measures ANOVA). FDR correction revealed that both between- and within-category (Bw+Wi) similarity increases best explained changing activity patterns in the superior frontal gyrus (SFG) and right temporal pole (rTP). The SFG also appeared to show increases in between-category similarity (Bw) and within-category similarity (Wi), and a decrease in within-category similarity of the CS- category. Interestingly, in all experimental phases, the lateral occipital cortex (LOC) preferred the semantic category model, and we did not find evidence for conditioning-based modulation within object-selective cortex. Error bars represent SEM.

\section{Lateral occipital cortex prefers the semantic model, regardless of experimental phase}

Given previously demonstrated pattern similarity changes in object-selective cortex, we wondered whether such regions would also yield similar results with respect to betweencategory similarity changes. However, the LOC showed a preference for the semantic model, which consistently outperformed the next-most-preferred model (the Wi model, which is a similar model [Spearman's $\rho \approx 0.76]$ ) at all three experimental phases $\left(\mathrm{Bl}: \mathrm{t}_{20}=2.97, \mathrm{p}_{\text {unc }}=0.0075 ; \mathrm{Co}: \mathrm{t}_{20}\right.$ $\left.=2.64, \mathrm{p}_{\text {unc }}=0.016 ; \mathrm{Ex}: \mathrm{t}_{20}=4.85, \mathrm{p}_{\text {unc }}=9.66 \times 10^{-5}\right)$. Unlike the SFG and ATL, there was not sufficient evidence to demonstrate that the $\mathrm{Bw}+\mathrm{Wi}$, the $\mathrm{Bw}$, or the $\mathrm{Wi}$ models were modulated by fear-conditioning $\left(\mathrm{t}_{20}=1.62, \mathrm{p}=0.120 ; \mathrm{t}_{20}=\right.$ $2.06, \mathrm{p}=0.053 ; \mathrm{t}_{20}=0.282, \mathrm{p}=0.781$, respectively) in the LOC. Despite the trend of the Bw model from the baseline phase to the conditioned phase, it is interesting to note that the Bw model tended to anticorrelate with activity patterns in the LOC, predominantly during the baseline and extinction phases $\left(\mathrm{t}_{20}=-2.94, \mathrm{p}_{\text {unc }}=0.008 ; \mathrm{t}_{20}=-2.68, \mathrm{p}_{\text {unc }}=0.015\right.$, respectively), but less so at the conditioned phase $\left(\mathrm{t}_{20}=-1.87\right.$, $\mathrm{p}_{\text {unc }}=0.076$ ).

\section{No evidence for relationship between anxiety scores and conditioning effects}

Using the STAI data that the participants provided, we Pearson correlated the STAI scores with the changes in 
correlation, between baseline and conditioned, for the $\mathrm{Bw}+\mathrm{Wi}, \mathrm{Bw}, \mathrm{Wi}$, and CS- models in the SFG and the $\mathrm{Bw}+\mathrm{Wi}$ model in the rTP (i.e., the models that survived the FDR-correction). Ultimately, we were unable to find such relationships between these variables, with the exception of a possible correlation between the Bw model and the SFG's correlation changes $(r=0.43, p=0.053)$. See Table 3 for a full list of the correlation results.

\section{Discussion}

This study aimed to investigate how information represented in the human brain is altered as a function of aversively conditioning exemplars from multiple semantic categories. Prior work combining fMRI and fear-conditioning has often focused on differences between a CS+ and CS-, either in terms of category representations [10] or generalization gradients [9, 28]. Conducting an experiment with three semantic categories (2 CS+'s and 1 CS-), we employed the framework of representational similarity analysis (RSA) [11] to test whether fear-conditioning would also generalize between categories and whether such between-category effects would also be present in the same brain regions in which within-category effects have been reported.

Our primary findings revealed that activity patterns within the SFG and the rTP-two regions previously reported to have shown pattern changes following aversive-learning [8, 24])—were best explained by the Bw+Wi model, indicating increased similarity of both between- and within-category exemplars after fear-conditioning. Such findings suggest that fear-generalization, as measured by the similarity of activity patterns, can extend beyond the level of single categories in certain brain regions, and that regions like the SFG and

Table 2. Descriptive statistics of the contrast Conditioned > Baseline for the five models at all six regions. ${ }^{*} p_{\text {unc }}<0.05,{ }^{* *} p_{F D R}<0.05$

\begin{tabular}{clrrrr} 
& & \multicolumn{1}{c}{ Mean } & SEM & \multicolumn{1}{c}{ T-score } & p-value \\
\cline { 2 - 6 } Amygdala & Bw+Wi & 0.010052 & 0.004747 & 2.117532 & $* 0.046939$ \\
& Bw & 0.006518 & 0.004396 & 1.482783 & 0.153719 \\
& Wi & 0.005508 & 0.0043 & 1.280972 & 0.214859 \\
& CS- & -0.00808 & 0.004934 & -1.63807 & 0.117046 \\
& Sem. Cat. & -0.00052 & 0.003535 & -0.14707 & 0.884552 \\
\hline \multirow{5}{*}{ Fusiform } & Bw+Wi & 0.004389 & 0.00785 & 0.560302 & 0.581496 \\
& Bw & 0.005209 & 0.005875 & 0.887166 & 0.385534 \\
& Wi & 0.000013 & 0.005555 & 0.00272 & 0.997857 \\
& CS- & -0.01158 & 0.005655 & -2.04756 & 0.053965 \\
& Sem. Cat. & -0.00768 & 0.004139 & -1.8545 & 0.078472 \\
\hline \multirow{5}{*}{ Insula } & Bw+Wi & 0.018375 & 0.00678 & 2.709709 & $* 0.013488$ \\
& Bw & 0.01592 & 0.004838 & 2.39547 & $* 0.0265$ \\
& Wi & 0.010396 & 0.004983 & 2.086404 & $* 0.049956$ \\
& CS- & -0.009 & 0.006021 & -1.49539 & 0.150426 \\
& Sem. Cat. & 0.00317 & 0.00347 & 0.913564 & 0.371829 \\
\hline \multirow{5}{*}{ LOC } & Bw+Wi & 0.020797 & 0.012771 & 1.624754 & 0.119871 \\
& Bw & 0.022005 & 0.010685 & 2.059107 & 0.052744 \\
& Wi & 0.002772 & 0.009743 & 0.281663 & 0.781096 \\
& CS- & -0.00832 & 0.014387 & -0.57322 & 0.572886 \\
& Sem. Cat. & -0.00308 & 0.009087 & -0.33974 & 0.737593 \\
\hline \multirow{5}{*}{ SFG } & Bw+Wi & 0.037394 & 0.006144 & 6.081204 & $* * 0.000006$ \\
& Bw & 0.027162 & 0.005128 & 5.295427 & $* * 0.000035$ \\
& Wi & 0.017542 & 0.005022 & 3.490896 & $* * 0.002303$ \\
& CS- & -0.02244 & 0.006357 & -3.52666 & $* * 0.00212$ \\
& Sem. Cat. & 0.000538 & 0.004407 & 0.121469 & 0.904532 \\
\hline \multirow{6}{*}{} & Bw+Wi & 0.021073 & 0.005264 & 4.003018 & $* * 0.000699$ \\
& Bw & 0.01238 & 0.004516 & 2.740872 & $* 0.012597$ \\
& Wi & 0.012847 & 0.004268 & 3.009954 & $* 0.006919$ \\
& CS- & -0.01544 & 0.005103 & -3.02523 & $* 0.006685$ \\
& Sem. Cat. & 0.001054 & 0.003664 & 0.28757 & 0.776635 \\
\hline & & & & &
\end{tabular}

rTP play a more general role in processing fear information (as our results imply an additional supracategorical effect in that not all participants were conditioned to the same two categories). This role may be intrinsically linked to semantic cognition, given that previous studies have related semantic processing to functional and structural properties of the anterior temporal lobes [29-31] as well as to multivoxel activity patterns in the SFG [32, 33]. Alternatively, these regions' role in fear-generalization may underlie affective processes, as diverse aspects of emotion information processing have been linked to both the SFG [34-37] and the anterior temporal lobe [38-40]. This second point is bolstered by the fact that the results we reported within the insula reflected those from the SFG and the rTP. Although our insular results did not surpass the FDRcorrected statistical thresholds, numerous studies have linked the insula to affective processing [41-47].

Activity patterns within the object-selective LOC were best explained by the semantic category model, regardless of the experimental phase. This observation of categorylevel representations within the LOC fits with prior literature $[48,49]$ and is also further supported by the finding that the $\mathrm{Bw}$ model (the only model containing no within-category similarity) was generally anticorrelated with LOC activity patterns (though this particular point results from uncorrected p-values, so we refrain from drawing conclusions based solely on these statistics). Interestingly, we did not find that activity patterns within object-selective cortex or the amygdala were modulated by fear-conditioning, which stands in contrast to previous findings [10]. However, we would not call this result a failure to replicate prior work because it is, in fact, unclear to what degree our results are directly comparable to those of previous reports, as the experimental paradigms differed. Specifically, our experiment resulted in two of three categories being aversively conditioned; thus, the majority of stimulus material would yield a higher shock expectancy, as opposed to one-half of stimulus material in studies that contain one CS+ category and one CS- category.

This aspect of the design brings up two points: first, the representations within the SFG and rTP may already abstract away from semantic categories altogether and instead reflect fear information. This idea is supported by the fact that we fear-conditioned two distinct semantic categories. As such, any brain regions that appear to reflect some form of between-category conditioning may be reflecting supracategorical or more generic information pertaining to the affective processes underlying the fear circuitry. Additionally, given that different participants were conditioned to different sets of categories, the group-level results we present add further evidence to this point. This interpretation is also in line with the previous finding of increased similarity between a semantically unrelated CS+ category and a phobic category in the rTP [8].

Second, investigations that employ more fear-conditioned than neutral categories may be studying processes in a fundamentally different state than those experiments 
bioRxiv preprint doi: https://doi.org/10.1101/2020.05.21.107805; this version posted May 25, 2020. The copyright holder for this preprint (which was not certified by peer review) is the author/funder, who has granted bioRxiv a license to display the preprint in perpetuity. It is made available under aCC-BY-NC 4.0 International license.

Table 3. Results from Pearson correlating the STAI scores with the changes in the FDR-corrected models' explanatory value. P-values are in parentheses.

\begin{tabular}{lcccc} 
& \multicolumn{2}{c}{ SFG } & \multicolumn{2}{c}{ rTP } \\
Bw+Wi & STAI-S & STAI-T & STAI-S & STAI-T \\
\cline { 2 - 5 } Bw & $0.113(0.627)$ & $-0.041(0.860)$ & - & - \\
Wi & $0.428(0.053)$ & $-0.217(0.344)$ & $0.357(0.112)$ & $-0.003(0.990)$ \\
CS- & $0.277(0.225)$ & $-0.285(0.211)$ & - & - \\
\hline
\end{tabular}

employing an equal number of fear-conditioned and neutral categories. This potentially distinction may result from participants internally normalizing the aversive nature of the "environment", thereby increasing the saliency or the value of the safe category at some cognitive level. In such cases, the information entropy [50] is lower, giving rise to potentially different physiological processing of threatrelated (un)certainty [51-54].

In summary, our experiment revealed increases in both between- and within-category similarity in more anterior regions of the brain: specifically, the superior frontal gyrus and the right temporal pole. Both of these areas have been previously implicated in diverse aspects of emotion information processing; as such, it is conceivable that these regions play a role in representing abstract information pertinent to fear-learning. This notion places a spotlight on such regions when translating related experimental designs to the clinical domain in order to explore potential functional imaging-related biomarkers of anxiety disorders.

\section{Acknowledgments}

The authors would like to thank Dasa Zeithamova-Demircan for contributing stimulus material. The formatting style for this manuscript derives from the "HenriquesLab bioRxiv template" by Ricardo Henriques, used under CC BY 4.0.

\section{Conflict of interest}

None declared

\section{Author contributions}

SL, MK, and JS designed the study. SL and MK acquired data, analyzed data, and drafted the manuscript. SL, MK, and JS revised the manuscript. RR contributed experiment resources. All authors approved the final version of the manuscript.

\section{Data availability}

The data that support the findings of this study are available from the corresponding author upon reasonable request.

\section{References}

1. Watson, J. B. and Rayner, R. Conditioned emotional reactions. Journal of Experimental Psychology, 3(1):1-14, 1920 doi: 10.1037/h0069608.

2. Fendt, M. and Fanselow, M. S. The neuroanatomical and neurochemical basis of conditioned fear. Neuroscience and Biobehavioral Reviews, 23(5):743-760, 1999. doi 10.1016/S0149-7634(99)00016-0.

3. Dymond, S., Dunsmoor, J. E., Vervliet, B., Roche, B., and Hermans, D. Fear Generalization in Humans: Systematic Review and Implications for Anxiety Disorder Research. Behavior Therapy, 46(5):561-582, 2015. doi: 10.1016/j.beth.2014.10.001.

4. Jasnow, A. M., Lynch, J. F., Gilman, T. L., and Riccio, D. C. Perspectives on fear generalization and its implications for emotional disorders. Journal of Neuroscience Research, 95(3):821-835, 2017. doi: 10.1002/jnr.23837.
5. Somers, J. M., Goldner, E. M., Waraich, P., and Hsu, L. Prevalence and Incidence Studies of Anxiety Disorders: A Systematic Review of the Literature. The Canadian Journal of Psychiatry, 51(2):100-113, 2006. doi: 10.1177/070674370605100206.

6. Fullana, M. A., Harrison, B. J., Soriano-Mas, C., Vervliet, B., Cardoner, N., Àvila-Parcet, A., and Radua, J. Neural signatures of human fear conditioning: an updated and extended meta-analysis of fMRI studies. Molecular Psychiatry, 21(4):500-508, 2016. doi: 10.1038/ mp.2015.88.

7. Haxby, J. V., Gobbini, M. I., Furey, M. L., Ishai, A., Schouten, J. L., and Pietrini, P. Distributed and overlapping representations of faces and objects in ventral temporal cortex. Science, 293(5539):2425-30, 2001. doi: 10.1126/science.1063736.

8. Levine, S. M., Pfaller, M., Reichenberger, J., Shiban, Y., Mühlberger, A., Rupprecht, R., and Schwarzbach, J. V. Relating experimentally-induced fear to pre-existing phobic fear in the human brain. Social Cognitive and Affective Neuroscience, 13(2):164-172, 2018. doi: $10.1093 / \mathrm{scan} / \mathrm{nsx} 147$.

9. Onat, S. and Büchel, C. The neuronal basis of fear generalization in humans. Nature Neuroscience, 18(12):1811-1818, 2015. doi: 10.1038/nn.4166.

10. Dunsmoor, J. E., Kragel, P. A., Martin, A., and LaBar, K. S. Aversive Learning Modulates Cortical Representations of Object Categories. Cerebral Cortex, 24(11):2859-2872, 2014. doi: $10.1093 /$ cercor/bht138

11. Kriegeskorte, N., Mur, M., and Bandettini, P. Representational similarity analysis connecting the branches of systems neuroscience. Frontiers in Systems Neuroscience, 2:1-28, 2008. doi: 10.3389/neuro.06.004.2008.

12. Zeithamova, D., de Araujo Sanchez, M.-A., and Adke, A. Trial timing and pattern-information analyses of fMRI data. Neurolmage, 153:221-231, 2017. doi: 10.1016/j.neuroimage.2017. 04.025 .

13. Brady, T. F., Konkle, T., Alvarez, G. A., and Oliva, A. Visual long-term memory has a massive storage capacity for object details. Proceedings of the National Academy of Sciences, 105 (38):14325-14329, 2008. doi: 10.1073/pnas.0803390105.

14. Konkle, T., Brady, T. F., Alvarez, G. A., and Oliva, A. Conceptual distinctiveness supports detailed visual long-term memory for real-world objects. Journal of Experimental Psychology: General, 139(3):558-578, 2010. doi: 10.1037/a0019165.

15. Schwarzbach, J. A simple framework (ASF) for behavioral and neuroimaging experiments based on the psychophysics toolbox for MATLAB. Behavior Research Methods, 43(4):11941201, 2011. doi: 10.3758/s13428-011-0106-8.

16. Brainard, D. H. The Psychophysics Toolbox. Spatial Vision, 10(4):433-436, 1997. doi: 10.1163/156856897X00357.

17. Laux, L., Glanzmann, P., Schaffner, P., and Spielberger, C. Das State-TraitAngstinventar-Manual. Beltz, Weinheim, Germany, 1981.

18. Setsompop, K., Gagoski, B. A., Polimeni, J. R., Witzel, T., Wedeen, V. J., and Wald, L. L. Blipped-controlled aliasing in parallel imaging for simultaneous multislice echo planar imaging with reduced g-factor penalty. Magnetic Resonance in Medicine, 67(5):1210-1224, 2012 doi: $10.1002 / \mathrm{mrm} .23097$.

19. Seidel, P., Levine, S. M., Tahedl, M., and Schwarzbach, J. V. Temporal Signal-toNoise Changes in Combined Multislice- and In-Plane-Accelerated Echo-Planar Imaging with a 20- and 64-Channel Coil. Scientific Reports, 10(1):5536, 2020. doi: 10.1038/ s41598-020-62590-y.

20. Smith, S. M., Jenkinson, M., Woolrich, M. W., Beckmann, C. F., Behrens, T. E., JohansenBerg, H., Bannister, P. R., De Luca, M., Drobnjak, I., Flitney, D. E., Niazy, R. K., Saunders, J., Vickers, J., Zhang, Y., De Stefano, N., Brady, J. M., and Matthews, P. M. Advances in functional and structural MR image analysis and implementation as FSL. Neurolmage, 23 (SUPPL. 1):S208-S219, 2004. doi: 10.1016/j.neuroimage.2004.07.051.

21. Oosterhof, N. N., Connolly, A. C., and Haxby, J. V. CoSMoMVPA: multi-modal multivariate pattern analysis of neuroimaging data in Matlab / GNU Octave. Frontiers in Neuroinformatics, 10:27, 2016. doi: 10.1101/047118.

22. Smith, S. Fast robust automated brain extraction. Human Brain Mapping, 17(3):143-155 2002. doi: 10.1002/hbm.10062.

23. Jenkinson, M., Bannister, P., Brady, M., and Smith, S. Improved Optimization for the Robust and Accurate Linear Registration and Motion Correction of Brain Images. Neurolmage, 17 (2):825-841, 2002. doi: 10.1006/nimg.2002.1132.

24. Visser, R. M., Scholte, H. S., and Kindt, M. Associative Learning Increases Trial-by-Trial Similarity of BOLD-MRI Patterns. Journal of Neuroscience, 31(33):12021-12028, 2011. doi: 10.1523/JNEUROSCI.2178-11.2011.

25. Frazier, J. a., Chiu, S., Breeze, J. L., Makris, N., Lange, N., Kennedy, D. N., Herbert, M. R., Bent, E. K., Koneru, V. K., Dieterich, M. E., Hodge, S. M., Rauch, S. L., Grant, P. E., Cohen, B. M., Seidman, L. J., Caviness, V. S., and Biederman, J. Structural Brain Magnetic Resonance Imaging of Limbic and Thalamic Volumes in Pediatric Bipolar Disorder. American Journal of Psychiatry, 162(7):1256-1265, 2005. doi: 10.1176/appi.ajp.162.7.1256.

26. Desikan, R. S., Ségonne, F., Fischl, B., Quinn, B. T., Dickerson, B. C., Blacker, D., Buckner, R. L., Dale, A. M., Maguire, R. P., Hyman, B. T., Albert, M. S., and Killiany, R. J. An automated labeling system for subdividing the human cerebral cortex on MR scans into gyral based regions of interest. Neurolmage, 31(3):968-980, 2006. doi: 10.1016/j.neuroimage.2006.01.021

27. Benjamini, Y. and Hochberg, Y. Benjamini Y, Hochberg Y. Controlling the false discovery rate: a practical and powerful approach to multiple testing. Journal of the Royal Statistical Society B, 57(1):289-300, 1995. doi: 10.2307/2346101.

28. Lissek, S., Bradford, D. E., Alvarez, R. P., Burton, P., Espensen-Sturges, T., Reynolds, R. C. and Grillon, C. Neural substrates of classically conditioned fear-generalization in humans: a parametric fMRI study. Social Cognitive and Affective Neuroscience, 9(8):1134-1142, 2014. doi: $10.1093 / \mathrm{scan} / \mathrm{nst} 096$.

29. Pobric, G., Jefferies, E., and Lambon Ralph, M. A. Amodal semantic representations depend on both anterior temporal lobes: Evidence from repetitive transcranial magnetic stimulation. Neuropsychologia, 48(5):1336-1342, 2010. doi: 10.1016/j.neuropsychologia. 2009.12.036.

30. Binney, R. J., Embleton, K. V., Jefferies, E., Parker, G. J. M., and Lambon Ralph, M. A. The Ventral and Inferolateral Aspects of the Anterior Temporal Lobe Are Crucial in Semantic Memory: Evidence from a Novel Direct Comparison of Distortion-Corrected fMRI, rTMS, 
bioRxiv preprint doi: https://doi.org/10.1101/2020.05.21.107805; this version posted May 25, 2020. The copyright holder for this preprint (which was not certified by peer review) is the author/funder, who has granted bioRxiv a license to display the preprint in perpetuity. It is made available under aCC-BY-NC 4.0 International license.

and Semantic Dementia. Cerebral Cortex, 20(11):2728-2738, 2010. doi: 10.1093/cercor/ bhq019.

31. Garcin, B., Urbanski, M., Thiebaut de Schotten, M., Levy, R., and Volle, E. Anterior Temporal Lobe Morphometry Predicts Categorization Ability. Frontiers in Human Neuroscience, 12: 36, 2018. doi: 10.3389/fnhum.2018.00036.

32. Devereux, B. J., Clarke, A., Marouchos, A., and Tyler, L. K. Representational Similarity Analysis Reveals Commonalities and Differences in the Semantic Processing of Words and Objects. Journal of Neuroscience, 33(48):18906-18916, 2013. doi: 10.1523/JNEUROSCI. 3809-13.2013.

33. Simanova, I., Hagoort, P., Oostenveld, R., and van Gerven, M. A. J. Modality-Independent Decoding of Semantic Information from the Human Brain. Cerebral Cortex, 24(2):426-434, 2014. doi: $10.1093 /$ cercor/bhs 324 .

34. Kesler/West, M. L., Andersen, A. H., Smith, C. D., Avison, M. J., Davis, C., Kryscio, R. J., and Blonder, L. X. Neural substrates of facial emotion processing using fMRI. Cognitive Brain Research, 11(2):213-226, 2001. doi: 10.1016/S0926-6410(00)00073-2.

35. McLellan, T., Wilcke, J., Johnston, L., Watts, R., and Miles, L. Sensitivity to posed and genuine displays of happiness and sadness: A fMRI study. Neuroscience Letters, 531(2): 149-154, 2012. doi: 10.1016/j.neulet.2012.10.039.

36. Terasawa, Y., Fukushima, H., and Umeda, S. How does interoceptive awareness interact with the subjective experience of emotion? An fMRI Study. Human Brain Mapping, 34: 598-612, 2011. doi: 10.1002/hbm.21458.

37. Gao, C., Weber, C. E., Wedell, D. H., and Shinkareva, S. V. An fMRI Study of Affective Congruence across Visual and Auditory Modalities. Journal of Cognitive Neuroscience, pages 1-12, 2020. doi: 10.1162/jocn_a_01553.

38. Thompson, S. A., Patterson, K., and Hodges, J. R. Left/right asymmetry of atrophy in semantic dementia: Behavioral-cognitive implications. Neurology, 61(9):1196-1203, 2003. doi: 10.1212/01.WNL.0000091868.28557.B8

39. Mathiak, K. A., Klasen, M., Weber, R., Ackermann, H., Shergill, S. S., and Mathiak, K. Reward system and temporal pole contributions to affective evaluation during a first person shooter video game. BMC Neuroscience, 12(1):66, 2011. doi: 10.1186/1471-2202-12-66.

40. Saarimäki, H., Ejtehadian, L. F., Glerean, E., Jääskeläinen, I. P., Vuilleumier, P., Sams, M., and Nummenmaa, L. Distributed affective space represents multiple emotion categories across the human brain. Social Cognitive and Affective Neuroscience, 13(5):471-482, 2018. doi: 10.1093/scan/nsy018.

41. Damasio, A. R., Grabowski, T. J., Bechara, A., Damasio, H., Ponto, L. L., Parvizi, J., and Hichwa, R. D. Subcortical and cortical brain activity during the feeling of self-generated emotions. Nature Neuroscience, 3(10):1049-1056, 2000. doi: 10.1038/79871.

42. Anders, S., Lotze, M., Erb, M., Grodd, W., and Birbaumer, N. Brain activity underlying emotional valence and arousal: A response-related fMRI study. Human Brain Mapping, 23 (4):200-209, 2004. doi: 10.1002/hbm.20048.

43. Corradi-Dell'Acqua, C., Hofstetter, C., and Vuilleumier, P. Felt and Seen Pain Evoke the Same Local Patterns of Cortical Activity in Insular and Cingulate Cortex. Journal of Neuroscience, 31(49):17996-18006, 2011. doi: 10.1523/JNEUROSCI.2686-11.2011.

44. Touroutoglou, A., Hollenbeck, M., Dickerson, B. C., and Feldman Barrett, L. Dissociable large-scale networks anchored in the right anterior insula subserve affective experience and attention. Neurolmage, 60(4):1947-1958, 2012. doi: 10.1016/j.neuroimage.2012.02.012.

45. Veit, R., Singh, V., Sitaram, R., Caria, A., Rauss, K., and Birbaumer, N. Using real-time fMRI to learn voluntary regulation of the anterior insula in the presence of threat-related stimuli. Social Cognitive and Affective Neuroscience, 7(6):623-634, 2012. doi: 10.1093/scan/nsr061.

46. Zaki, J., Davis, J. I., and Ochsner, K. N. Overlapping activity in anterior insula during interoception and emotional experience. Neurolmage, 62(1):493-499, 2012. doi: 10.1016/ j.neuroimage.2012.05.012.

47. Levine, S. M., Wackerle, A., Rupprecht, R., and Schwarzbach, J. V. The neura representation of an individualized relational affective space. Neuropsychologia, 120:3542, 2018. doi: 10.1016/j.neuropsychologia.2018.10.008.

48. Walther, D. B., Caddigan, E., Fei-Fei, L., and Beck, D. M. Natural Scene Categories Revealed in Distributed Patterns of Activity in the Human Brain. Journal of Neuroscience, 29(34):10573-10581, 2009. doi: 10.1523/JNEUROSCI.0559-09.2009.

49. Iordan, M. C., Greene, M. R., Beck, D. M., and Fei-Fei, L. Basic Level Category Structure Emerges Gradually across Human Ventral Visual Cortex. Journal of Cognitive Neuroscience, 27(7):1427-1446, 2015. doi: 10.1162/jocn_a_00790.

50. Shannon, C. E. A Mathematical Theory of Communication. Bell System Technical Journal, 27(3):379-423, 1948. doi: 10.1002/j.1538-7305.1948.tb01338.x.

51. Grillon, C., Baas, J. P., Lissek, S., Smith, K., and Milstein, J. Anxious responses to predictable and unpredictable aversive events. Behavioral Neuroscience, 118(5):916-924, 2004. doi: 10.1037/0735-7044.118.5.916.

52. Dunsmoor, J. E., Bandettini, P. A., and Knight, D. C. Impact of continuous versus intermittent CS-UCS pairing on human brain activation during Pavlovian fear conditioning. Behavioral Neuroscience, 121(4):635-642, 2007. doi: 10.1037/0735-7044.121.4.635.

53. Somerville, L. H., Wagner, D. D., Wig, G. S., Moran, J. M., Whalen, P. J., and Kelley, W. M. Interactions Between Transient and Sustained Neural Signals Support the Generation and Regulation of Anxious Emotion. Cerebral Cortex, 23(1):49-60, 2013. doi: 10.1093/cercor/ bhr373.

54. Bradford, D. E., Kaye, J. T., and Curtin, J. J. Not just noise: Individual differences in general startle reactivity predict startle response to uncertain and certain threat. Psychophysiology, 51:407-411, 2014. doi: 10.1111/psyp.12193. 